ISSN: 2349-2031

Research Article

\title{
Le couvert forestier en Côte d'Ivoire : une analyse critique de la situation de gestion des forêts (classées, parcs et réserves)
}

\author{
Traore Kassoum \\ Université Péléforo Gon Coulibaly de Korhogo (Côte d'Ivoire),UFR des sciences sociales/Département de \\ Sociologie
}

\begin{abstract}
Preserving the forest is a "vital" action for maintaining the climate balance and promoting eco-tourism. The history mentions that the Ivorian forests played an important role in the dynamics of the development of the country because they sheltered a great diversity floristic and wildlife. Unfortunately, from 16 million hectares at the beginning of the last century (1900), the dense rainforest increased to 9 million hectares in 1965 and 3 million hectares in 1991. Today, it is estimated at about 2.5 million hectares. This situation is attributable to extensive agriculture based on the technique of shifting cultivation, the overexploitation of the forest with timber and wood energy and bush fires. Moreover, the phenomenon of forest infiltration with regard to the occupation or colonization of forest areas, particularly in the southwest of the country by migrants to build houses and / or villages, is an important factor in the disappearance of the forest. Also, successive crises that the country has experienced since 1999 until 2010 (post-election), with periods of tension, have led to mass displacements of populations. This regrouping of the populations in turn caused the clearing of the forest areas for their relocation. Thus, from the analysis of the situation of forest pressure in Côte d'Ivoire, the Ministry in charge of the management of the Ivorian forest cover indicates that the accelerated rate of destruction of Ivorian forests has today reached an estimated average of $\mathbf{4 5 0 , 0 0 0}$ hectares a year. The forest situation is catastrophic, if corrective measures are not taken and put into effect, the forest cover is supposed to disappear around 2040. The present work attempts to contribute to the level of reflection undertaken to improve the management of the sector.
\end{abstract}

Résumé: Préserver la forêt est une action « vitale " pour le maintien de l'équilibre climatique et la promotion de l'écotourisme. L'histoire mentionne que les forêts ivoiriennes ont joué un rôle important dans la dynamique du développement du pays car elles abritaient une grande diversité floristique et faunique. Malheureusement, de 16 millions d'hectares au début du siècle dernier (année 1900), la forêt dense humide est passée à 9 millions d'hectares en 1965 et à 3 millions d'hectares en 1991. Aujourd'hui, elle est estimée à environ 2,5 millions d'hectares. Cette situation est imputable à l'agriculture extensive basée sur la technique des cultures itinérantes sur brûlis, la surexploitation de la forêt en bois d'œuvre et bois énergie et les feux de brousse. Par ailleurs, le phénomène de l'infiltration des forêts au regard de l'occupation ou la colonisation des zones forestières notamment du Sud-Ouest du pays par des migrants pour bâtir les habitations et/ou des villages est un facteur important de la disparition du couvert forestier.Egalement, les crises successives qu'a connues le pays depuis 1999 jusqu'en 2010 (post-électorale), populations. Ce regroupement des populations à son tour, a occasionné le défrichement des zones forestières pour leur relocalisation. Ainsi, de l'analyse de la situation des pressions sur les forêts en Côte d'Ivoire, le ministère en charge de la gestion du couvert forestier ivoirien indique que le rythme accéléré de destruction des forêts ivoiriennes a atteint de nos jours une moyenne estimée à 450.000 hectares par an. La situation des forêts est catastrophique, si des mesures correctives ne sont pas entreprises et mises en vigueur, le couvert forestier doit en principe disparaitre vers 2040. Le présent travail tente d'apporter sa contribution au niveau des réflexions engagées pour améliorer la gestion du secteur.

Mots clés : forêts classées, parcs et réserves, gestion du couvert forestier, Côte d'Ivoire

Key Words: Classified forests, parks and reserves, forest cover management, Ivory Coast

\section{INTRODUCTION:}

Jadis, le couvert forestier ivoirien estimé à 16 millions d'hectares dans les années 1900 est aujourd'hui réduite à environ 2,5 millions d'hectares. Plusieurs raisons expliquent cette disparition et/ou diminution de la forêt en Côte d'Ivoire. En effet, l'agriculture a été et demeure encore un levier important dans le développement économique et humain du pays. Au lendemain de son accession à la souveraineté nationale, le pays a structuré sa croissance sur celle-ci en plus de l'industrialisation et de l'urbanisation. Pour atteindre cet objectif, de vastes étendues de forêts ont été défrichées pour créer d'immenses plantations des cultures de rente que sont le café, le cacao, le palmier à huile, l'hévéa, l'ananas, etc., et autres fruits tropicaux destinés à l'exportation.
En réalité, le développement de l'agriculture s'est caractérisé par un rythme aussi rapide de déboisement ${ }^{1}$ et a eu des répercussions sur le couvert forestier qui est estimé aujourd'hui à 7,8\% de la superficie totale du pays (Document Stratégie de Réduction de la Pauvreté). De sorte qu'une facette des problèmes environnementaux dont fait face le pays se caractérise par la déforestation.

En outre, la croissance démographique constitue un facteur important dans la perte des forêts ivoiriennes. De 10,8 millions

\footnotetext{
${ }^{1}$ Les statistiques estiment le déboisement à 500000 hectares/an entre 1960 et 1991.
} 


\section{Traore Kassoum / Le couvert forestier en Côte d'Ivoire : une analyse critique de la situation de gestion des forêts (classées, parcs et réserves)}

d'habitants en 1988 la population ivoirienne est passée à 15,3 millions d'habitants en 1998. Aujourd'hui, le dernier recensement général de la population et de l'habitat, estime à 22.671.331 millions d'habitants (RGPH, 2014). Cette croissance démographique rapide avec un taux d'accroissement annuel moyen de 2,55\% est l'une des plus élevées de l'Afrique. En plus, de l'accroissement naturel de la population, le phénomène de migration à la recherche d'un bien-être social et les mouvements des populations liés à des crises ou non, ont contribué fortement au peuplement du pays. Ainsi, cette forte croissance démographique constitue l'une des causes des pressions d'origine humaine -sous toutes ses formes $^{2}$ - qui s'exerce sur les ressources forestières d'une part et sur les terres agricoles d'autre part.

Par ailleurs, la détérioration de la situation économique durant ces trois décennies a davantage accentué les problèmes environnementaux notamment la perte vertigineuse des forêts entrainant le phénomène de désertification dans certaines régions du pays. Les conséquences néfastes qui en résulte ont contribué à accentuer la pauvreté et remis en cause, le processus de développement durable au regard de l'exploitation abusive des ressource forestières. Cette situation peut trouver son explication par le fait qu'à l'époque l'Etat Ivoirien dans sa stratégie de croissance économique, la sauvegarde de la forêt n'avait pas été explicitement mentionnée de façon formelle dans ce processus.

Heureusement, depuis le sommet de la Terre en 1992, il est apparue une prise de conscience dans le processus du développement tant au niveau des décideurs que des organisations de la société civile et des autres acteurs de la vie nationale. Sur cette base, les programmes de développement qui ont été élaboré jusque-là devraient tenir compte de l'exigence d'une gestion durable de l'environnement et des ressources naturelles parmi lesquelles la forêt. Face à la diminution au fil des années des ressources forestières du pays, de nombreuses interrogations légitimes au niveau national sont posées.

Comme réponse nationale et pour mieux intégrer toutes les dimensions environnementales, dans les différentes politiques et stratégies sectorielles, le pays a lancé depuis 2002, le processus du document Stratégie de Réduction de la Pauvreté (DSRP) pour un diagnostic sectoriel avec un accent particulier sur la situation de la forêt en Côte d'ivoire. Ce tableau sombre du statut du couvert forestier ivoirien en état de disparition, interpelle toutes les parties prenantes à un changement de comportement afin d'éviter ce cercle vicieux non reluisant pour le développement économique, social et humain du pays.

Ce présent travail vise à contribuer aux différentes réflexions et/ou débats déjà amorcés sur la problématique de la destruction et/ou disparition des forêts ivoiriennes en mettant l'accent sur la dynamique de l'approche de la gestion du couvert forestier en Côte d'Ivoire.

\footnotetext{
${ }^{2}$ Pratiques agricoles, urbanisation, feux de brousses, exploitation forestière abusive, élevage, etc.
}

Sur le plan méthodologique, le travail privilégie l'approche qualitative basée sur une analyse critique axée sur la revue documentaire, des discours relatifs à la situation de gestion des forêts ivoiriennes au regard du bilan des activités, des observations à partir des résultats des études réalisées dans le secteur.

Le travail présente dans un premier temps la question des défis majeurs de préservation des forêts ivoiriennes, ensuite la situation du couvert forestier ivoirien, puis le cadre de gestion $\mathrm{du}$ secteur et enfin les enjeux et perspectives pour une gestion durable du couvert forestier ivoirienne

\section{I- QUESTION DES DEFIS MAJEURS DE PRESERVATION DES FORETS IVOIRIENNE}

Le patrimoine forestier ivoirien ou le couvert forestier ivoirien traverse un moment difficile de son histoire qui a toujours été une richesse pour le pays. Les agressions dont il souffre aujourd'hui laissent à imaginer que si les forêts ne vont pas disparaitre dans les deux décennies avenirs? Les analyses faites de la situation des forêts indiquent que toutes les stratégies prises jusque-là pour les remettre en état n'ont pas atteint l'objectif fixé. Les différents massifs qui constituent ce couvert forestier continuent de subir la dégradation, à travers les multiples actions anthropiques dont les pratiques extensives de l'agriculture. En réalité, dans l'entendement ou dans l'esprit des populations, ces cultures de rente ou pérennes, notamment le cacao et le café ne peuvent être pratiquées que dans les forêts. Toutes ces cultures se font au détriment des nombreuses forêts. Malgré, les actions de mobilisation sociale à l'endroit des différentes générations de producteurs des cultures de rente en général et la sensibilisation menée auprès des producteurs de cacao et de café du pays pour les convaincre du contraire, ceux-ci demeurent attachés à cette croyance, qui a valeur de tradition dans leurs pratiques agricoles. Comme résultat, les forêts classées ivoiriennes ne le sont plus que de nom pour la plupart, car presqu'elles toutes renferment aujourd'hui non seulement de grandes plantations de cacao mais aussi les habitations des populations voire même les campements et villages. Une culture de grande importance pour l'économie nationale dont la production a augmenté ces deux dernières années, passant de 1,200 millions de tonnes à environ 1,700 millions de tonnes dans la campagne 2015-2016. A telle enseigne, certains spécialistes du domaine s'interrogent sur « la mort » programmée des forêts classées.

Pour faire face à la recrudescence des infiltrations et/ou agressions des forêts classées, depuis 2007, les gouvernements successifs à travers la Société de Développement des Forêts $\left(\right.$ SODEFOR $\left.^{3}\right)$ ont initié comme stratégie la contractualisation pour gérer cette situation de sorte à maintenir et développer le couvert forestier dans ces massifs classés, sans toutefois détruire pour autant les plantations de cacao. L'expérience lancée avait pour période de pilotage 2007-2009 avec pour la

\footnotetext{
${ }^{3}$ Société de développement des plantations forestières
} (Sodefor), une société étatique avec un mode de gestion privé 
phase pilote les forêts classées de la Brassué à Abengourou (située à l'est du pays) et de Irobo à Tiassalé (située au sud du pays). Ce projet dans son fonctionnement a rencontré des difficultés à s'étendre pour diverses raisons. Cependant, malgré les problèmes qu'a connus cette expérience dans sa mise en œuvre, les premiers résultats obtenus ne mettent pas en cause son efficacité ou n'autorisent pas sa mise systématique à l'écart. Elle demeure toujours appréciée de nombreux spécialistes du domaine.

Face aux défis liés à la gestion des forêts en Côte d'Ivoire, pourquoi ne pas poursuivre cette expérience par entremises d'un nouveau programme qui de toute façon devrait prendre en compte les faiblesses et les erreurs du passé en se dotant des moyens adéquats, indispensables à sa réussite? Aussi fautil mentionner qu'avec les réalités financières actuelles qui sont les siennes, la SODEFOR ne saurait efficacement conduire toute seule ce programme sans ressources additionnelles conséquentes? Les compétences humaines et l'expérience requise pour une telle action sont encore présentes à tous les niveaux de la chaine de gestion du patrimoine forestier. Il ne reste que la volonté manifeste et soutenue des pouvoirs publics à en faire une priorité nationale, au même titre que d'autres programmes.

Un autre défis lié à la gestion des forêts en Côte d'Ivoire, se situé au niveau du changement fondamental perpétuel que l'on a toujours constaté dans l'administration forestière ivoirienne, dès qu'un nouveau responsable prend la gestion de la filière, sans souvent tenir compte des acquis enregistrés. Dans ce contexte, cela ne peut que conduire à faire inévitablement du sur-place. Ainsi, à l'analyse, l'on est tenté de penser que cette manière de procéder semble bien servir certains intérêts assez particuliers. Mais, malheureusement qui ramène toujours à cette éternelle question relative à la protection du patrimoine forestier ivoirien : «pourquoi ça ne marche toujours pas?»

La question du défi présenté qu'en est-il de la situation du couvert forestier ivoirien?

\section{II- SITUATION DU COUVERT FORESTIER IVOIRIEN}

La Côte d'Ivoire fait partie de ces pays forestiers africains qui ont très tôt compris, l'urgence et la nécessité de conserver certains massifs forestiers du pays pour diverses raisons dont l'une très importante est d'assurer les équilibres climatologiques régionaux, favorisant les conditions écologiques appropriées à une bonne pratique agricole. Cependant, après cinquante (50) ans de vie de la nation qu'en est-il de la situation des forêts au fils des années ?

\section{II-1- Situation des forêts classées}

Les forêts classées sont l'une des catégories du domaine forestier de l'Etat qui comprend entre autres: les forêts protégées; les périmètres de protection et les reboisements. Les forêts classées consiste en un règlement de l'espace ou périmètre déterminé, délimité et aborné que l'on souhaite conserver en l'état pour des raisons diverses. Il est donc interdit l'exercice de certains droits d'usage portant sur le sol forestier, cause des dégradations, c'est le cas des défrichements.

Conscient de l'importance des biens faits des forêts, en Côte d'Ivoire, plusieurs périmètres ou domaines ont été classées. Les premiers classements de forêts remontent au cours des années 1920. A l'époque le domaine forestier classé de l'Etat était répartit sur sensiblement 218 massifs forestiers et couvrait une superficie d'environ 3.995.200 hectares sur toute l'étendue de territoire dont $70 \%$ en zone forestière et préforestière et $30 \%$ en zone de savane (SODEFOR).

Les statistiques indiquent qu'en 1960, le pays comptait 234 forêts classées d'une superficie globale d'environ 4,2 millions d'hectares qui aujourd'hui sont estimées à 231 forêts classées couvrant une superficie globale environ 4 millions d'hectares (Ministère Eaux et Forêts, 2016). Parmi celles-ci, il ne reste plus que 1,3 millions d'hectares de forêts encore non dégradée selon la SODEFOR. Cependant, malgré des ressource limitées, les responsables de la SODEFOR estiment avoir doté à $61 \%$ des forêts classées du pays depuis 1993, d'un plan d'aménagement et réalisé le reboisement de 210.000 hectares de forêts en 50 ans. Toutefois, le bilan de la gestion du couvert forestier est mitigé. De plus, la SODEFOR a perdu la quasitotalité de son acquis en raison de la crise socio-politicomilitaire de 2002 à 2011, qui a secoué et divisé en deux le pays. Selon les experts de la SODEFOR, plus de deux millions d'hectares de forêts, soit 113 forêts classées qui étaient situées en zone contrôlée par la rébellion au Nord, ont été pillées par une exploitation anarchique, créant des dégâts importants (Communication SODEFOR, septembre 2016).

Cela dénote du niveau de dégradation de nos forêts. Il faut noter que cette situation de perte à grande vitesse des forêts ivoiriennes n'est pas un phénomène récent. Depuis des décennies l'alerte avait été lancée et les statistiques estimaient en 1997 cette dégradation à 30\% de la superficie totale des forêts classées des zones forestières et pré forestières comprenant 149 massifs pour environ 2.715.000 hectares.

Pourtant, les droits d'usage portant sur les fruits et les produits forestiers sont limités. Il s'agit du ramassage de bois morts, de la cueillette des fruits et des plantes alimentaires ou médicinales, de l'exploitation des bois d'industrie et de service, des parcours des animaux. Dans ces forêts classées, l'exploitation est subordonnée à la délivrance d'un permis d'exploitation indiquant les conditions de cette exploitation (cahier des charges). Toutes ces dispositions sont prises dans l'optique de pérenniser l'existence des forêts car les forêts jouent un rôle important dans l'évolution des sols, de la faune, de la flore, du cycle de l'eau, de l'équilibre du milieu et même de la production agricole. De nos jours, la caractéristique essentielle actuelle de ces forêts classées est la succession de formations végétales souvent associées : forêt dense fermée, forêt dense ouverte, forêt dégradée, association forêt-culture, cultures, friches et jachères.

Les résultats de l'étude réalisée (2009) par Société française de réalisation d'étude et de conseil (Sofreco) par système de 
télédétection des forêts classées en Côte d'Ivoire entre 1998 et 2008 révèlent une véritable dégradation de celles-ci. Les forêts fermées de certains domaines forêts classées auraient même disparu avec l'évolution du temps. Il s'agit des forêts de Go Bodiénou (61 642 ha), Irobo (40 864 ha), Mopri (32 300 ha), Téné (30 036 ha).

Ces mêmes statistiques indiquent également que sur l'ensemble des forêts classées sous la gestion de la SODEFOR, seulement une soixantaine (60) de forêts classées en-dessous du 8ème parallèle dispose d'un plan d'aménagement rédigé dont une dizaine en application ou exécution. La plupart des forêts classées au nord du pays sont délimitées sur des plans mais pas ou partiellement délimitées sur le terrain.

Comment se présentent les parcs et réserves sous la gestion de l'Etat?

\section{II-2- Situation des parcs et réserves}

En Côte d'Ivoire, le gouvernement a fait des efforts considérables pour préserver ses principaux écosystèmes naturels en mettant en place un large réseau de parcs et de réserves.

$\mathrm{Au}$ fils des années, avec les agressions qu'elles continuaient de subir, ces aires protégées sont passées à environ 1,9 million d'hectares, soit $6 \%$ de la superficie totale du pays (Banque Mondiale Infos, Numéro 5). Les principaux et les plus grands parcs sont :

- la Comoé avec 1,2 million hectares,

- le Taï avec 330.000 hectares,

- la Marahoué avec 101.000 hectares,

- le Mont Sangbé avec 95.000 hectares.

Il faut préciser que les réserves de Taï et de la Comoé ont été déclarés sites naturels de l'héritage universel sous la convention internationale concernant la protection de l'Héritage Culturel et Naturel Universel et sont reconnues comme réserves de biosphères dans le programme de l'UNESCO/MAB ${ }^{4}$.

Cependant, ces sites (parcs et réserves) subissent une pression très importante des populations à tel enseigne que le gouvernement éprouve du mal à protéger de manière efficace leur intégrité. Cependant, il faut noter que certain site tel que la réserve de la Comoé reste encore raisonnablement moins dégradé, mais celles de Taï et de la Marahoué sont beaucoup infiltrées de parcelles agricoles et autres activités humaines. Ces sites sont aussi confrontés ou souffrent d'un sérieux pratique de braconnage. A l'analyse, il n'est pas alarmant de

\footnotetext{
4 Le programme pour Man and Biosphère (MAB) est l'un des cinq programmes de l'Unesco dans le domaine des sciences exactes et naturelles. Le programme MAB propose un projet interdisciplinaire dont le but est d'améliorer les relations entre les gens et leur environnement global. La volonté de ce programme est de mettre à contribution la recherche scientifique pour la réduction de la perte de biodiversité, ceci par des approches écologiques,
}

dire que les lois protégeant la faune sauvage et les parcs sont difficiles à faire observer et à appliquer parce que l'administration de gestion éprouve des difficultés à traduire les contrevenants devant les tribunaux. Cette situation est aggravée par le manque et/ou l'insuffisance d'infrastructures adéquates dans les parcs et réserves.

Les études réalisées sur les forêts ivoiriennes indiquent dans le même sens des résultats non reluisants pour celles-ci. Selon l'Union Internationale pour la Conservation de la Nature (UICN), la surface totale des du réseau (aires protégées) s'estimait à 2,10 millions hectares, soit $6,5 \%$ du territoire en 2008. Ce réseau se constitue principalement de huit (08) parcs nationaux, de trois (03) réserves de faune et trois (03) réserves naturelles intégrales avec respectivement une superficie de 1 828574 ha, de 236130 ha et de 7.500 ha. Il existe également deux (02) réserves de faune-flore et dix-sept (17) réserves botaniques.

$\mathrm{Au}$ regard du bilan et des résultats d'évaluation, la situation des parcs et réserves a été jugée alarmante par l'UICN, car ces aires protégées ont connu beaucoup de dégradation et d'infiltrations. A titre d'exemple, en 2008 le seul parc de la Marahoué abriterait plus de 49000 cultivateurs selon l'UICN. Ces données confirment les résultats de l'étude de télédétection réalisée par Sofreco qui indiquent que les forêts de certaines aires protégées, auraient même quasiment disparu, c'est le cas de la Marahoué ou du Mont Péko (Sofreco, 2009).

Qu'en est-il des causes et les contraintes qui font obstruction à la protection des forêts ?

\section{II-3- Cause et Contraintes à la protection des aires protégées}

\section{II-3-1 Cause de la déforestation}

Du diagnostic de la situation des forêts en Côte d'Ivoire, comme déjà souligné dans l'introduction, l'on note les causes directes et indirectes de la disparition des forêts. Notre étude n'ayant pas la prétention de faire l'analyse exhaustive de celles-ci présente quelques-unes.

Concernant les causes directes : le secteur agricole constitué des cultures de rentes et des cultures vivrières ont été et sont responsables de la perte d'une grande partie du patrimoine forestier ivoirien imputable seulement aux surfaces plantées des cultures de rentes. Toutes ces cultures accompagnées par les grands programmes de développement agricole en cours comme le programme national d'investissement agricole (PNIA) sont des voies anticipatoires pour la disparition des forêts attribuables au cacao (18\%), à l'Hévéa ( $9 \%$ ), au palmier à huile $(5 \%)$, au riz $(48 \%)$ et à l'igname (13\% à 42\%). Il faut noter que les plantations de café et de cacao, dont la superficie a quintuplé en moins de 50 ans, sont des causes principales de la déforestation. En outre, les données techniques indiquent également que la pratique de ces différentes spéculations mentionnées sont généralement itinérantes et annuellement consommatrices de fertilité forestière ou de jachères. Cela fait de l'agriculture le plus puissant « moteur » de la déforestation 
en Côte d'Ivoire.

Face à cette situation aussi alarmante de la disparition des forêts, le premier ministre du gouvernement ${ }^{5}$ en 2014 affirmait que: "Plus des trois quarts (3/4) des forêts de la Côte d'Ivoire ont disparu en un demi-siècle du fait de l'agriculture extensive et de l'urbanisation. Il mentionne que la forêt ivoirienne a connu une "dégradation continue", passant de "16,5 millions d'hectares à l'indépendance" en 1960 à "deux millions aujourd'hui", soit "moins de $13 \%$ du territoire national contre 78\%". La déforestation et la mise en place d'une "agriculture extensive et l'urbanisation accélérée" ont largement contribué à cette "dégradation", a déploré le premier ministre. Le gouvernement a mis en place une politique de "gestion durable et rationnelle des ressources forestière" afin de ramener la "couverture du territoire audessus du seuil international de 20\%", a-t-il évoqué »

Ces propos tenus se justifient à partir des réalités des faits évoqués par la Société ivoirienne de développement des plantations forestières (SODEFOR) qui évaluait à 300.000 hectares la superficie de forêts disparaissant chaque année du fait de "l'exploitation abusive" du bois, liée à la production de charbon ou à des coupes d'arbres pour l'exportation d'essences précieuses. La déforestation est également provoquée par des feux de brousse incontrôlés déclenchés par des agriculteurs, qui dégagent ainsi de l'espace pour de nouvelles cultures de cacao, dont la Côte d'Ivoire est le premier producteur mondial. Certaines forêts classées, à l'instar du Parc national du Mont Peko (Ouest) ou de celui de la Maraoué (centre) ont ainsi presque complètement disparues du fait de la culture du cacao.

Il va plus loin en ces termes, "L'agriculture assure à elle seule "plus de $30 \%$ du PIB, $70 \%$ des recettes d'exportation et occupe plus $60 \%$ de la population active"» et "que les statistiques agricoles indiquent que quelque 1,74 million de tonnes de cacao ont été récoltées durant la campagne 2013 2014, un record historique pour la Côte d'Ivoire. Outre le cacao, la noix de cajou, dont le pays est deuxième producteur mondial, a connu une production record cette année. "

Il faut signaler également que les pratiques d'élevage et l'exploitation de gisements miniers jouent un rôle non moins important dans la perte du couvert forestier ivoirien. Malgré, la promotion de l'élevage intensif, l'élevage reste traditionnel et extensif pour les populations. De nos jours, plusieurs espaces sont aménagés pour la pratique de l'élevage afin d'assurer l'autosuffisance alimentaire. De ce point de vu, on peut considérer que l'élevage a un impact sur la perte des forêts même s'il semble être modéré. Concernant, l'exploitation des minéraux, le pays dispose d'un important gisement d'or, bauxite, magnésium, nickel, etc. L'extraction de ces minéraux engendre inéluctablement l'expansion des agglomérations et le développement des infrastructures telles les routes, les habitations, les grands centres ou services sociaux de base. Ces interventions au profit de l'économie impliquent la destruction des forêts.

\footnotetext{
${ }^{5}$ le premier ministre ivoirien Daniel Kablan Duncan
}

En ce qui concerne les causes indirectes: l'un des phénomènes le plus visible est la pression démographique liée aux vagues successives migratoires des populations et à l'accroissement naturel. En effet, l'installation des communautés issues de grands mouvements migratoires vers la Côte d'Ivoire a permis l'occupation et/ou la colonisation des zones forestières pour des objectifs agricoles depuis l'accession à l'indépendance du pays. La politique agricole prônée à l'époque en faveur des cultures de rente notamment le binôme café-cacao, a conduit aux déplacements aussi bien des populations du nord du pays et les ressortissants des pays voisins vers les zones forestières comme main d'œuvre agricole. Après des décennies passées, la réduction et l'appauvrissement des terres cultivables sous l'influence conjuguée de la croissance démographique élevée et d'une agriculture trop traditionaliste ont entrainé aussi un important afflux de populations allogènes et allochtones. Certaines populations vivant dans des conditions précaires se sont déplacées à la recherche de reliques de forêts localisées généralement dans les régions du Sud et de l'Ouest du pays. Ce qui a pour corollaire les nombreux conflits fonciers dans les zones d'accueil.

En effet, les conflits liés aux problèmes fonciers n'est pas un phénomène nouveau mais ils se sont beaucoup aggravés surtout pendant les périodes de crises successives que le pays a connu depuis l'évènement du coup d'état de 1999 à 2010 période postélectorale. Aujourd'hui encore, les conflits de cette nature demeurent et représentent un obstacle à la cohésion sociale et la gestion des forêts ivoiriennes. Au nombre des manifestations, l'on note que des dynamiques rurales, parfois contradictoires, se sont croisées et ont conduit la région forestière, notamment à l'Ouest, dans un état de forte confusion sur l'appropriation des terres, et qui persiste encore malgré les actions de médiation. Ce regroupement des populations du fait des conflits se caractérisent par le retour des autochtones, le déplacement des populations affectées par les crises et l'occupation des nouvelles terres par des nouveaux arrivants.

Les effets de l'insécurité dans cette partie sud-ouest du pays pendant les périodes de crises socio-politiques avec des manifestations de conflits armés, ont eu un impact considérable sur la destruction des forêts. Les données de certaines études ont révélé que les communautés allogènes installées (notamment Burkinabés) dans la forêt classée de Mont Péko qui a totalement disparu depuis 2008. Plusieurs milices d'autodéfenses sont toujours actives dans la région de l'Ouest précisément les départements de Guiglo et Duekué. Dans cette situation incontrôlée, l'on constate des acteurs non professionnels qui sont érigés en exploitant du bois (milices, rebelles, mercenaires, populations locales, etc.), car la filière s'est révélée comme une source de financement utile pour l'effort de guerre ou tout simplement pour s'enrichir.

Par ailleurs, le manque de sécurisation foncière a permis l'installation ou l'occupation anarchique des forêts par des populations ivoiriennes et étrangères. En effet, les pratiques 
administratives coutumières ont longtemps contredire le cadre juridique. Selon Chauveau (2009), l'accès des terres de manière informelle par la pratique administrative coutumière a permis à certaines catégories acteurs notamment les hommes politiques d'influencer la redistribution ou la distribution des droits concernant le domaine coutumier. Cette situation a permis d'enregistrer beaucoup de transactions foncières. Si la loi foncière de 1998 avait pour objectif de conforter les paysans sur leurs terres en leur octroyant des titres d'occupation, il convient de souligner néanmoins que peu de domaine était cédé, c'est le droit coutumier qui avait en charge le reste. Les résultats de l'étude de Sofreco (2009) indique que seulement $2 \%$ des terres du domaine foncier rural fait 1 'objet d'un titre de propriété délivré par l'administration. Par conséquent, cette loi censée résoudre le problème de la concurrence foncière, mais conduite au détriment d'une partie de la population, n'a fait qu'envenimer ou empirer une situation sociale déjà tendue, avec la survenue d'un débat national sur l'«ivoirité».

Le dysfonctionnement au niveau des structures est une cause indirecte. En effet, l'instabilité institutionnelle du secteur depuis l'indépendance de pays (1960) représente une cause dans la mauvaise gestion du patrimoine forestier. En réalité, l'arrimage de l'administration des eaux et forêts à plusieurs ministères avec les changements de stratégies privilégiant tantôt coercition, tantôt la concertation n'ont fait qu'affaiblir le pouvoir contractuel des institutions de gestion surtout de la SODEFOR vis-à-vis des paysans et communautés installés dans les forêts classées et limiter les résultats obtenus au niveau des déguerpissements. Cette situation renvoie au défaut de pilotage et aux insuffisances de la tutelle du fait du nonapport de correctifs. En outre, le manque de consensus et l'insuffisance de concertation entre les différents acteurs sont un problème crucial dans la gestion du secteur. Le constat est que les différentes administrations se concertent faiblement ou pas. Ce qui fragilise encore la lutte contre les exploitants véreux et les populations clandestins qui infiltrent les forêts classées, les parcs et réserves. Ce déficit est préoccupant parce que les mécanismes interinstitutionnels présents ne permettent pas de fixer le cadre suffisamment précis. Ce cloisonnement de travail rend inefficace les stratégies de lutte déforestation.

Ainsi, au-delà de causes énumérées qui font obstruction à la gestion des forêts, il existe également des contraintes.

\section{II-3-2 Contraintes liées à la protection des forêts}

Les difficultés liées à la protection du couvert forestier ivoirien sont diverses et multiformes. Cette partie essaie de faire une analyse non exhaustive de ces contraintes. En effet, le Ministère des Eaux et forêts, premier responsable en charge de la gestion du patrimoine forestier en collaboration avec les structures de la protection de la Nature des ministères de la Salubrité, de l'Environnement et du Développement Durable, des Ressources Animales et Halieutiques, de l'Agriculture et du Développement Local, malgré un enthousiasme certain, sont confrontées à une insuffisance de ressources humaines, d'équipement et des ressources financières pour assurer la protection efficace de ces aires protégées. La loi protégeant les forêts classées, les parcs, réserves et la faune sauvages sont difficiles à faire observer et appliquer car l'administration éprouve des difficultés à traduire les exploitants véreux de ces aires devant les tribunaux.

$\mathrm{Au}$ niveau des contraintes financières, l'étude de Hallé et Bruzon (2006) indique que la part du budget de l'état alloué à la gestion de l'environnement en général tourne autour de $0,8 \%$ du budget national, ce qui reste très faible. Ce taux aussi faible du budget révèle le manque d'une réelle volonté politique en faveur de l'environnement encore moins de la gestion de couvert forestier. Le soutien financier des bailleurs de fonds quoique important ne suffisent pas de gérer tous les aspects de la gestion des forêts. Généralement ces fonds sont orientés à des volets bien précis en fonction des projets. Aussi, faut-il insister encore sur l'instabilité institutionnelle car l'administration des eaux et forêts à plusieurs fois été arrimée à d'autres ministères. Ce changement de tutelle ne favorise pas la mise en œuvre des stratégies de lutte contre la dégradation des domaines forestiers protégés.

En outre, le manque d'infrastructures adéquates dans les parcs et réserves aggrave cette situation car l'on note l'occupation anarchique avec un niveau d'agression aussi alarmante de ces domaines protégés. On peut noter également l'absence ou 'insuffisance des textes clairs dans les différents plans d'aménagement du territoire relatifs à l'utilisation des terres. Et même quand ils existent, non seulement ils ne sont pas suffisamment traités et leur application demeure hypothétique face à la mauvaise gouvernance (délinquance et corruption). Les résultats du diagnostic sur les forêts et la pauvreté en Côte d'Ivoire en 2002 indiquent les nombreux cas de défrichements illicites, d'exploitation forestière frauduleuse, de fausses déclarations de volumes et de la qualité des bois constituent autant de facteurs négatifs pour une gestion durable de la forêt.

A ce propos, l'Union Internationale pour la conservation de la Nature (2008) mentionnait que seulement cinq parcs avaient des plans d'aménagement et de gestion à l'exception la forêt de Taï pour indiquer la faiblesse à moyenne de la gestion. De même à part les îles Ehotilé et du parc du Banco, les communautés ne sont pas impliquées dans la gestion des aires protégées. Par ailleurs, ces plans manquent le plus souvent de mesures en direction des populations car disent-ils « après décret ministériel de classement, les droits d'usage des communautés locales n'ont quasiment jamais été purgés par l'Etat et les indemnités compensatrices n'ont jamais été payées, d'où les conflits permanents avec les communautés locales dans quasiment tous les parcs et réserves»

Le défi de l'autosuffisance alimentaire notamment en matière de la riziculture représenterait une contrainte pour la préservation des forêts. En plus des cultures de rente destructives des forêts déjà connu, la satisfaction de la totalité de la demande locale de riz par la production nationale ainsi que la constitution d'un stock de sécurité et l'exportation du surplus de production pourrait avoir un possible impact négatif sur les forêts, car demande l'aménagement de beaucoup de 
terres pour atteindre cet objectif. Les statistiques de l'étude ${ }^{6}$ réalisée sur les forêts ivoiriennes en 2013, indiquent que la production 2018 serait de 3,25 millions de tonne par an de paddy sur 1,38 millions hectare, avec un rendement moyen de 2,35 tonnes par hectare. Sur cette base, la mise en place de ce plan pourrait entrainer la disparition de près de $48 \%$ de la couverture forestière actuelle. A partir des projections du document Stratégie Nationale de Développement de la Riziculture (SNDR), les statistiques ont prévu qu'en 2016 la satisfaction des besoins en riz blanchi serait 1,9 millions de tonne par an et qu'en 2018 elle serait 2,1 millions de tonne par an et en stock de sécurité de 0,2 millions de tonne par an. Avec un surplus d'environ 10\% (estimation prévu pour 2018), l'estimation totale serait de 2,92 millions de tonne de riz blanchi en 2030. Ce scénario serait peu crédible car la mise en culture annuelle de plus de 4 millions hectares de riz pluvial, dont $9 \%$ sur la forêt, entraînerait le déboisement de plus de 360000 hectares par an et la disparition de l'ensemble des forêts ivoiriennes en moins de 10 ans.

Enfin, les formations pour ne pas dire les élites politiques influencent beaucoup et négativement la gestion et/ou la protection des forêts. A ce niveau, la contrainte majeure se situe dans les relations que celles-ci ont avec les populations. Pendant que certains groupements politiques luttent pour la protection des forêts en prenant des mesures, entre autres de délocalisation ou le retrait des infiltrés des forêts d'autres groupements politiques activent la haine entre les différentes communautés et entre les populations et les gouvernants. Cette situation de méfiance entre les populations rend difficile la gestion et ou le suivi des interventions de lutte contre l'occupation anarchique et abusive des forêts.

Qu'en est-il de la situation du fonctionnement du secteur forestier ivoirien?

\section{III- CADRE DE GESTION DU COUVERT FORESTIER EN COTE D'IVOIRE}

L'analyse du cadre de gestion du couvert forestier ivoirien s'intéresse non seulement à la dynamique de gestion du secteur à travers les différentes structures mises en place dans son organisation mais aussi de l'engagement de l'Etat ivoirien comme réponse aux conventions ratifiées sur la sauvegarde des forêts.

\section{III-1- Instabilité de l'Administration forestière}

De l'analyse de l'évolution de la gestion des forêts ivoiriennes, le secteur a longtemps été influencé dans son fonctionnement par une instabilité institutionnelle. Les structures administratives en charge de la gestion des activités forestières et environnementales ont connu une instabilité quasi-permanente depuis son accession à l'indépendance en

\footnotetext{
${ }^{6}$ Dans le cadre du Programme de coopération des Nations Unies pour la réduction des émissions dues au déboisement et à la dégradation des forêts dans les pays en développement (ONU-REDD)
}

1960. L'arrimage de ces structures à d'autres ministères, s'accompagne toujours avec le changement systématique des noms de celles-ci. Dans son évolution, on est parti de la Direction des Eaux et Forêts à la Direction de la Police Forestière de 1960 à 1966; puis la Direction des Eaux et Forêts et de la Chasse de 1966 à 1970; pour aboutir au Secrétariat d'Etat chargé de la reforestation et des parcs nationaux de 1971 à 1974.

Le premier Département ministériel pour les Eaux et Forêts est créé en 1974; à partir de cette date, cette instabilité va se renforcer davantage jusqu'à l'heure actuelle. Ainsi, à partir de 1976, les différentes activités relevant du secteur forestier seront confiées à deux ministères: le Ministère des Eaux et Forêts et le Ministère de la Protection de la Nature. Jusqu'en 1977.

En février 1981, deux Ministères se partagent les activités. Il s'agit du Ministère des Eaux et Forêts et du Ministère de l'Environnement. En novembre 1983, le Gouvernement à l'époque tout en associant les activités forestières et agricoles, crée le Ministère de l'Agriculture et des Eaux et Forêts en confiant la pisciculture et la pêche au Développement Rural. En juillet 1986, le Ministère des Eaux et Forêts retrouve sa place au sein du nouveau gouvernement. Les changements se sont toujours poursuivis et en 1990 est né le Ministère de l'Agriculture et des Ressources Animales.

A partir de l'an 2000, les différents gouvernements sont passés successivement du Ministère de la Construction et de l'Environnement, du Ministère de l'Environnement de l'Eau et de la Forêt pour aboutir enfin au Ministère des Eaux et Forêts et Ministère du Cadre de vie et de l'Environnement en 2001. Après la longue crise d'une décennie, c'est-à-dire après la crise post-électorale en 2010, le gouvernement actuel de la $3^{\text {ème }}$ république redonne la gestion du secteur de plein droit et de plein exercice au Ministère des Eaux et Forêts.

Malgré tous ces problèmes relevés, dans son fonctionnement, la diversité des activités forestières a amené l'Administration forestière à créer des structures plus ou moins spécialisées. Cette spécialisation s'est opérée dans quatre grands domaines à savoir:

- la production de bois et l'industrie forestière

- la protection de la nature, de l'environnement et de l'exercice de la chasse

- la pêche et la pisciculture

- la formation et la recherche forestière

Ces instabilités ont eu pour conséquences : (i) un sérieux handicap dans le contrôle, le suivi et même la poursuite des projets et programmes initiés dans le secteur, (ii) l'insuffisance de la mise en œuvre de certaines réformes qui auraient dû non seulement apporter un changement structurel, mais aussi une autonomie fonctionnelle pour apporter une réponse aux problèmes de gestion du secteur. A cela, il faut noter le personnel technique en nombre insuffisant et moins expérimenté, des moyens matériel et financier inexistants. En 
dépit de toutes ces contraintes, le pays dispose néanmoins d'une administration opérationnelle qui essaie de mettre en œuvre des politiques et stratégies de gestion des forêts. (Etude sur l'environnement, la forêt et la pauvreté en Côte d'Ivoire).

Les réalités ou les difficultés liées à la gestion du couvert forestier a conduit l'Etat de Côte d'Ivoire à s'engager au plan international dans le cadre de la préservation de celui-ci. Qu'en est-il de l'engagement de l'Etat vis-vis des conventions internationales relatives à la préservation des forêts?

\section{III-2- Engagement de l'Etat dans la préservation des forêts ivoiriennes.}

Conscients des problèmes environnementaux qui longtemps se sont signalés dans le pays, en particulier la diminution du couvert forestier, les différents gouvernements qui se sont succédés au fil des années ont eu une gouvernance par l'engagement de faire de la bonne gestion des ressources naturelles et de protection de l'environnement, une question centrale dans la stratégie de lutte contre la pauvreté. Cette prise de conscience a été constaté tant au niveau des décideurs que des organisations de la société civile et des autres acteurs de la vie nationale.

Dans cette perspective d'idée, la Côte d'Ivoire a démarré son plan d'action environnemental dans un contexte favorable marqué par l'organisation de la conférence des Nations Unies sur l'environnement et le développement (Rio de Janeiro, Brésil 3-4 juin 1992). Le processus préparatoire de ce plan dénommé Plan National d'Action pour Environnement de Côte d'Ivoire (PNAE-CI) a vu l'élaboration d'un "livre blanc de l'environnement" qui fut adopté en novembre 1996. Ce programme Action 21 ou Agenda 21 reflète un consensus mondial et un engagement politique au niveau le plus élevé sur la coopération en matière de développement et d'environnement.

En signant la déclaration finale de Rio, la Côte d'Ivoire confirme son adhésion aux principes et aux objectifs du développement durable auxquelles se rattachent des Conventions internationales, entre autres la Convention cadre des Nations Unies sur le Changement Climatique (CCNUCC), la convention sur la Diversité Biologique (CDB), etc.

Mais avant, le gouvernement a pris en 1994, des dispositions pour améliorer son rang dans la conservation au niveau international en ratifiant la Convention sur le Commerce International des espèces menacées $\left(\mathrm{CITES}^{7}\right)$, la Convention Ramsar sur les zones humides et la Convention sur la diversité biologiques de United Nations Conference on Environment and Development (UNCED). Ces engagements ont été soutenus par la mise la mise en place du Plan National d'Actions de l'Environnement (PNAE) dans lequel est comprise une stratégie de la protection de la nature. En collaboration avec le soutien du Fond pour l'Environnement Mondial (FEM), cette stratégie est élaborée et s'articule autour de trois grands domaines d'intervention : (a) la protection des parcs et réserves existants; (b) la promotion des ressources sauvages en dehors des sites protégés; (c) et l'amélioration des connaissances biologiques grâce au développement des programmes de recherches. L'engagement de l'état pour sauver le patrimoine forestier a permis l'élaboration d'une stratégie de gestion des parcs nationaux et réserves en 1995 avec l'appui de l'Union Internationale pour la Conservation de la Nature (UICN).

Au plan de la gouvernance, l'engagement de l'état se traduit par la Toujours création et le maintien d'un ministère en charge des Eaux et Forêts même si celui-ci a été plusieurs fois arrimé à d'autres ministères à cause de l'instabilité administrative. Ainsi, l'engagement de l'Etat à travers le Ministère en charge des Eaux et Forêts se matérialise depuis 1968 à travers les différents codes forestiers qui ont existé dont le dernier adopté en date de 2014 qui est censé inverser la tendance actuelle. La nouvelle loi crée un cadre de "gestion durable de la forêt" et introduit des innovations en matière de conservation et de reconstruction du couvert végétal avec "une plus grande implication des populations".

Ces engagements en faveur de la gestion du couvert forestier ivoirien ne peuvent être une réalité sans développer des partenariats pour le fonctionnement du secteur.

\section{III-3- Partenariat développé par l'Etat pour la préservation des aires protégées}

Dans le cadre de la mise en œuvre de sa politique forestière, le développement d'un partenariat est devenu une voie capitale pour un accompagnement dans la gestion des forêts classées, parcs et réserves sous le contrôle de l'état. Ainsi, la communauté des bailleurs de fonds ont apporté un appui technique et financier considérable dans le domaine de la conservation des forêts et aires protégées en Côte d'Ivoire. A ce niveau, on peut citer entre autres la coopération Allemande GIZ (ex GTZ/KWF), la coopération Canadienne (ACDI), le Haut Commissariat pour les Réfugiés (HCR) qui ont apporté et continue pour certains d'apporter leur appui. A ceux-là, il y a la Banque Mondiale, la BAD, etc. Cependant, bon nombre d'entre eux ont manifesté leur intérêt pour le financement d'activités spécifiques ou des projets dans le cadre de la politique forestière. Ainsi, plutôt que de renforcer la capacité du pays à gérer les parcs et les réserves, ils ont orienté leurs interventions sur le développement de sites spécifiques (Banque Mondiale Info, numéro 5).

Ce partenariat a conduit le Gouvernement ivoirien a déployé d'énormes efforts pour garantir la conservation des principaux écosystèmes naturels par la création d'un réseau de parcs et de réserves. Selon les estimations de la Banque Mondiale ces aires protégées sont estimées à 1,9 millions d'hectares soit $6 \%$ de la superficie totale du pays. Elles comprennent les plus grands parcs et réserves que sont la Comoé, le Taï, la Marahoué, le Mont Sangbé (voir la situation des parcs et réserves plus haut).

${ }^{7}$ Convention on International Trade of Endangered Species 
Ces actions en faveur des parcs et réserves, ont permis d'ériger les aires protégées de Taï et de la Comoé comme sites naturels du patrimoine mondial de l'Unesco ou de l'héritage universel sous la convention internationale relative à la protection de l'Héritage Culturel et Naturel Universel. Toutefois, la réalité sur le terrain révèle que les parcs de Taï et de la Marahoué sont infiltrés et font face à une pression anthropique aussi importante avec des parcelles agricoles par endroit.

Ainsi, dans le cadre du partenariat, avec le soutien de la Banque Mondiale, le Plan National de Développement de la Forêt (PNDF) pour la période de 1988 à 2015 est élaboré. Ce plan à long terme visait d'une part à protéger les ressources des forêts naturelles restantes et à améliorer leur gestion, et d'autre part à préserver les avantages produits des aires protégées et à assurer la contribution à long terme du secteur de la forêt dans l'économie ivoirienne. Plusieurs programmes et/ou projets ont été exécuté grâce au partenariat développé dans le secteur, entre autres, le Projet Sectoriel Forestier (PSF) intitulé prêt 3186 IVC avec pour objectif de promouvoir la conservation et la gestion durable des Forêts Tropicales Humides 8 existantes. Les résultats sont édifiants car la mise en œuvre de ce projet a permis : (i) de renforcer les capacités humaines et techniques de la SODEFOR principale agence d'exécution du gouvernement, (ii) d'élaborer une politique nationale de planification et de gestion de l'interface population/forêts, (iii) à faire l'inventaire des forêts et de la biodiversité sur une superficie de 900.000 hectares, (iv) à protéger les forêts classées contre de nouvelles infiltrations clandestines sur environ plus de 1,9 million d'hectares, (v) à réduire l'exploitation forestière non contrôlée sur l'ensemble des forêts classées du pays.

Cette expérience de la Banque Mondiale a été significative en développant une telle approche de partenariat. Son soutien s'est montré comme un élément catalyseur pour d'autres partenariats tels que le Projet de Conservation et de Gestion des Aires Protégées (PCGAP), le Projet National de Gestion du Terroir et de l'Equipement Rural (PNGTER) ${ }^{9}$ en lien direct avec le Projet National d'Appui aux Services Agricoles (PNASA).

En guise d'illustration de l'importance de ce partenariat dans la gestion du couvert forestier, le ministre en charge des Eaux et Forêts de la 3eme république de la Côte d'Ivoire lançait un plaidoyer auprès du gouvernement et des partenaires au développement afin qu'ils soutiennent le fonctionnement du secteur car selon lui «Beaucoup de nos hommes travaillent au péril de leur vie. Nous perdons chaque année des agents qui sont confrontés à des bandes armées et à des trafiquants qui $n$ 'hésitent pas à tuer. Nous devons protéger nos agents afin qu'ils exercent mieux leur devoir de protection de la forêt »,

\footnotetext{
${ }^{8} \mathrm{TMF}=$ Tropical Moist forest

${ }^{9}$ PNGTER dont l'objectif principal est d'intensifier le niveau de vie de la population rurale de la Côte d'Ivoire en aidant à créer des conditions viables et des mécanismes d'exécution efficace pour le développement et la gestion durable des ressources naturelles et de l'infrastructure rurale.
}

Aujourd'hui encore, dans la mise en œuvre du nouveau code forestier, l'appui des partenaires au développement est nécessaire selon le ministère en charge des Eaux et Forêts. Parce que le cadre juridique désormais bien défini, il ne reste que les moyens financiers pour la mise en œuvre des grands axes de développement et de promotion de la forêt qui passent par une intensification de la politique de reboisement. A preuve, ce sont les partenaires techniques qui ont apporté le financement pour l'organisation des états généraux de la forêt qui s'est tenu en Mai 2015 à hauteur de 100 millions FCFA (communication, Ministère des Eaux et Forêts, février 2015). Il est important de rappeler que 380 mille hectares de forêt ont été reboisés entre 1929 et 2014 avec leur soutien.

En plus des bailleurs de fonds, le partenariat s'étend auprès des opérateurs de la filière ${ }^{10}$ qui contribuent à la reconstitution $\mathrm{du}$ couvert forestier par la réalisation de leur quota de reboisement calculé sur la base de 1 ha de reboisement pour $205 \mathrm{~m} 3$ de bois exploité en zone forestière ou $150 \mathrm{~m} 3$ de bois exploité en zone pré-forestière. Ici, l'opérateur de la filière doit réaliser au prix coûtant en collaboration avec ou sous contrôle de la SODEFOR, un certain nombre de travaux d'aménagement convenus chaque année d'accord parties.

Les Organisations non gouvernementales (ONG) et les associations de la société civile ont un rôle prépondérant aussi bien en amont qu'en aval par l'identification, la recherche, la sensibilisation, la conception et l'exécution des projets initiés par l'administration technique. Par exemple, pour ne citer que l'Ong SOS-forêt et l'observatoire ivoirien pour la gestion durable des ressources naturelles (OI-Ren) qui se sont mobilisées pour contester contre la décision gouvernementale de 2017 de transformation des forêts classées en «agroforêt ». A l'analyse, ils estiment que «maintenir en permanence les agriculteurs dans les forêts classées sera un feu vert pour la disparition et/ou la mort programmé des forêts ivoiriennes ».

A l'analyse, le partenariat demeure un canal essentiel et même privilégié pour la mise en œuvre des plans stratégies ou de l'exécution de la politique forestière parce qu'il permet de veiller à une implication de toutes les parties prenantes.

$\mathrm{Au}$ regard de cette grande mobilisation sociale autour des forêts classées, parcs et réserves qui a aboutir la naissance d'un nouveau code forestier, que prévoit les nouvelles stratégies de gestion du couvert forestier en Côte d'Ivoire ?

\section{ENJEUX ET PERSPECTIVES POUR UNE GESTION DURABLE DU PATRIMOINE FORESTIER IVOIRIEN}

A partir des analyses faites sur la disparition et/ou la dégradation du couvert forestier, les problèmes au niveau de la forêt ivoirienne s'articulent autour : du recul du couvert forestier, la perte de la biodiversité, dégradation des sols

\footnotetext{
${ }^{10} \mathrm{Ce}$ sont les exploitants et les industriels forestiers, les exploitants agricoles, les coopératives forestières
}

(communication, Ministère des Eaux et Forêts, février 2015). 


\section{Traore Kassoum / Le couvert forestier en Côte d'Ivoire : une analyse critique de la situation de gestion des forêts (classées, parcs et réserves)}

cultivables, changement climatique et désertification. Face à un tableau sombre qui dépeint la perte vertigineuse du patrimoine forestier ivoirien, la révision des textes relatifs à la gestion du secteur s'est avérée nécessaire. Ainsi, le nouveau code forestier promulgué en juillet 2014 présente les enjeux et les perspectives d'une gestion durable du couvert forestier ivoirien. En d'autres termes, la nouvelle loi crée un cadre de "gestion durable de la forêt" et introduit des innovations en matière de conservation et de reconstruction du couvert végétal avec "une plus grande implication des populations".

Pour rappel, il faut noter que plusieurs écrits et rapports sur le secteur estiment que la situation du couvert végétal est « catastrophique ». Aujourd'hui, le pays ne possède qu'à peine 2 millions hectares de forêts. les raisons de cette destruction à grande échelle sont connues. Il s'agit de l'exploitation forestière à des fins commerciales, les feux de brousse et l'agriculture, etc. Les statistiques indiquent un taux de déforestation d'environ 200.000 hectares par an, et les projections estiment que la forêt ivoirienne doit en principe disparaitre vers 2040 si des mesures correctives ne sont pas entrées en vigueur.

C'est dans cette perspective que les différentes réflexions qui ont eu lieu après la mise en place du Gouvernement au lendemain de la crise post-électorale 2010, estimaient que, la situation de la disparition de la forêt ivoirienne est aggravée par l'inadaptation du premier code forestier de 1965 et qui aujourd'hui est obsolète. Selon les responsables du secteur, à l'époque « la solution pour les décideurs dès les années 80 a consisté à reboiser. Cependant, les objectifs d'environ 300.000 hectares à reboiser chaque année n'ont jamais été atteints. Et que le pic de 10.000 hectares par an n'ayant jamais été dépassé ». C'est au vu de toutes ces défaillances, que le Gouvernement a porté la loi N²014-du 14 juillet 2014 définissant le nouveau code forestier ivoirien. Selon eux, ce nouveau code opère un virage important par rapport à l'ancien dans le sens où il prend toutes les dispositions pour inverser la tendance actuelle. Il corrige plusieurs insuffisances relevées dans l'ancien code.

En effet, la nouvelle loi forestière, dispose les règles relatives à la gestion durable des forêts. Spécifiquement, elle vise à : (i) renforcer la protection de la forêt; (ii) préserver et valoriser la diversité biologique et contribuer à l'équilibre des écosystèmes forestiers; (iii) favoriser la constitution des taux de couverture forestière représentant au moins $20 \%$ de la superficie du territoire national.

Aux dires des responsables en charge de la gestion du secteur, les raisons sont multiples avec des enjeux importants. Le couvert forestier se raréfiant, cela occasionne de nombreux conflits et provoque l'infiltration d'éléments dans les espaces protégés. Sans détour et sans la langue de bois, le Ministre en charge des Eaux et forêts mentionne en ces termes: " l'exemple du mont Péko avec une majorité d'exploitants illégaux venant du Burkina Faso est là pour nous édifier. Aujourd'hui, l'on constate la recrudescence des conflits fonciers entre allogènes et étrangers ou entre autochtones et allogènes, etc. qui se multiplient et menacent la cohésion entre les populations». C'est pourquoi, cette nouvelle loi est une opportunité, puisqu'elle souligne la primauté de l'Etat sur les forêts classées, les aires protégées et permet d'engager des actions vigoureuses afin de solutionner ces crises qui apparaissent un peu partout.

Un enjeu aussi capital du nouveau code forestier est le rétablissement de la cohésion sociale et le vivre ensemble autour des forêts, car personne n'ignore que la longue crise qu'a connue le pays a favorisé de multiples infractions. Sur cette base, il a estimé que les recommandations des états généraux tenus en mai 2015 et rendus publics permettront de faire la part des choses et de favoriser l'application du nouveau code forestier.

En somme, le nouveau code forestier est le principal 'outil" d'inversion des tendances au triple plan "écologique, économique et éducatif". Selon les responsables du secteur, 'la mise en œuvre du nouveau code permettra aux générations présentes et futures ainsi qu'aux amoureux de la nature de découvrir, d'apprendre et de mieux connaitre notre écosystème par la préservation de sa biodiversité". Toutefois, le nouveau code ne signifie pas un abandon de la gestion des activités forestière par l'Etat qui se réserve 'les fonctions du choix et de l'encadrement des opérateurs, du suivi et du contrôle des activités, de la réglementation, de la formation et de la recherche".

\section{CONCLUSION}

Aux termes de notre analyse sur la situation de gestion du couvert forestier ivoirien, il ressort que la quasi-totalité des forêts classées de Côte d'Ivoire est en voie de destruction en raison des occupations illégales et des défrichements sauvages. Autrement dit, cette situation est imputable aux "activités anthropiques" notamment, l'agriculture extensive basée sur la technique des cultures itinérantes sur brûlis, la surexploitation de la forêt en bois d'œuvre et bois d'énergie, les feux de brousse, les infiltrations et/ou l'occupation anarchique ainsi que les divers aléas climatiques dont la sécheresse. Ce qui laisse comprendre que « la situation du couvert végétal est catastrophique »

Les premières solutions fondées sur le reboisement jusque-là en cours ne représentent qu'à peine $4 \%$ "des pertes de forêt estimées à 200.000 hectares par an". Pour seulement environ 380.000 hectares qui ont été reboisés pour la période de 1929 à 2014 avec l'implication de la société civile aux côtés de l'Etat (Communication, Ministère de Eaux et Forêts, février 2015). Sur cette base, les projections estiment qu'à ce rythme "il n'y aura plus de forêt en 2040 en Côte d'Ivoire". L'analyse révèle aussi que même la journée nationale qui a été instituée en juillet 1983 dans le but de sauvegarder la forêt, en proie aux dangers a connu ses limites pour plusieurs motifs dont l'épine dorsale est la question de financement du secteur.

Néanmoins, le pays a déployé et continue de faire beaucoup d'efforts pour la restauration du couvert forestier non seulement avec le potentiel interne par la création des 
ministères en charge de l'Environnement, des Eaux et Forêts mais aussi avec l'accompagnement des partenaires au développement. C'est ainsi qu'avec le soutien des bailleurs de fonds, le pays dispose des capacités techniques pour agir sur la réduction de la perte et/ou la disparition des forêts ivoiriennes prenant en compte l'amélioration de la gestion transversale et participative de l'environnement. Le nouveau code forestier adopté depuis 2014 illustre bien ce partenariat entre le gouvernement, les partenaires au développement et les auteurs de la vie nationale.

C'est sur cette base que le pouvoir public affirme que 'la mise en cuvre du nouveau code permettra aux générations présentes et futures ainsi qu'aux amoureux de la nature de découvrir, d'apprendre et de mieux connaitre notre écosystème par la préservation de sa biodiversité". Le pays a besoin d'élaborer et de mettre en place un modèle de parc national qui prenne en compte les impacts socio-économiques de l'exclusion de production directe dans les sites protégés et atténuer les conflits avec les populations périphériques et certaines autorités locales.

Si la Côte d'Ivoire se fixe pour but de parvenir à une société dont les citoyens sont sensibilisés à la question du développement durable sous toutes ses dimensions et qui possèdent les connaissances nécessaire, il faut dans ce cas développement la collaboration entre les parties car aucun individu, aucun groupe, aucun gouvernement ne peut régler à lui seul la question du développement durable en particulier la gestion du couvert forestier

\section{REFERENCES BIBLIOGRAPHIES}

[1] Académie Nationale des sciences 1992. Une planète, un avenir, nouveaux horizons, USA, 187p 1992

[2] Alain S. 1993. Rapport sur les domaines et actions prioritaires en matière d'éducation environnementale : aspect formation, PNAE-CI, 1993

[3] $\mathrm{BAD}, 1990$. politique en matière d'environnement, 1990,61p

[4] Banque Mondiale, 1995. Environnement, « la biodiversité en Côte d'Ivoire », bulletin trimestriel d'information de la mission régionale de l'Afrique de 1'Ouest, $\mathrm{N}^{\circ} 5$, Juin 1995.

[5] Comité de supervision du DSRP, 2002. Etude sur l'environnement, la forêt et la pauvreté en Côte d'Ivoire $70 \mathrm{p}$

[6] Côte d'Ivoire, 2002. Etude sur l'environnement, la forêt et la pauvreté en Côte d'Ivoire, Comité de supervision du DSRP, Cabinet du premier ministre, 2002

[7] FMI- Banque Mondiale, 1993: Fiances et développement « faire durer le développement », publication trimestrielle, décembre 1993, 53p

[8] FNUAP, 1989. Prévenir l'avenir 41p

[9] Fonds de partenariat pour le carbone forestier (FPCF)
(2013) : Programme de coopération des Nations Unies pour la réduction des émissions dues au déboisement et à la dégradation des forêts dans les pays en développement (ONU-REDD), RCI, Novembre 2013 Page

[10]Hennu K., 1995. Le visage Humain de l'environnement africain, environnement et politique sociale, séries de document de travail, ESP-15, Aout 1995

[11]Lester R. Brown et al, 1996. L'état de la planète, worldwatch institute, nouveaux horizons, $275 \mathrm{p}$, paris

[12] Loi portant code de l'environnement ivoirien, 1996

[13]Ministère des Eaux et Forêts, 2016. Rapports de communications sur le couvert forestier en Côtes d'Ivoire, 2016

[14] Observateur OCDE, 1989. environnement, climat, agriculture, $\mathrm{n}^{\circ} 156$ février 1989, 32p

[15] SODEFOR, 2016. Infiltration des forêts classées de Côte d'Ivoire : Réalités et solutions, Document de Conférence dans le cadre du lancement officiel de l'opération déguerpissement des forêts classées de Côte d'Ivoire, initiée par la Croix verte de Côte d'Ivoire, 20p

[16] Martine Tahoux T. 1998. Cours d'écologie humaine, université Abobo-Adjamé, Année 1997-1998. 